\title{
EFFECT OF PERSONAL BRANDING EFFORTS ON JOB PROSPECTS OF A LINKEDIN USER
}

\author{
Arghyadeep Basu \\ SIBM Bengaluru, SIU, Electronic City Phase-1, Bengaluru.
}

\begin{abstract}
In this article, the authors examined the relation between Personal Branding efforts in LinkedIn and their job prospects. While there are other social media sites which can be taken into consideration for the study, LinkedIn is deemed to be a purely professional social networking site with content relevant to jobs, industry, economy etc. The authors found that sizable LinkedIn users do not post, share or visit content with the intent of "Building Personal Brand", however wishes to attract the attention of potential recruiters on the platform. While the term "Personal Branding has been discussed in numerous papers, journals and articles, broader clarity is guaranteed on the same. Additionally, it was duly noted that a person looking for a job doesn't trust LinkedIn entirely and thus signs up on various other established job portals or seek suitable referrals at the same time.
\end{abstract}

"Your brand has moved online. That means you need to translate who you are in the real world into a congruent bits-and-bytes version of you. First impressions are important to your career success, so whether they take place with a handshake or on a handheld device, you need to make sure yours is authentic, compelling, and relevant.", said Mitch Joel in his book 'Ctrl, Alt, Delete'.

"It is much more than a style of dress, a particular degree or credential, or the associations to which you belong. Mainly it is the unique "stamp" you put on everything you say or do. Because no one will do things and present themselves in exactly the same way that you do, your uniqueness is what people notice"- Annabelle Reitman and Caitlin Williams on Personal Branding in their periodical journal 'Distinguish yourself and excel in the profession'.

Author Neal Schaffer in his journal "LinkedIn or Left Out" shares his view on LinkedIn by stating- "It is much more than a style of dress, a particular degree or credential, or the associations to which you belong. Mainly it is the unique "stamp" you put on everything you say or do. Because no one will do things and present themselves in exactly the same way that you do, your uniqueness is what people notice."
Further legal trainer Avery Black feels "The most effective way to start your job search is by developing relationships with people. The Internet, through professional websites like LinkedIn and social media sites like Face-book and Twitter, allows people to start a dialogue with each other."

The above quotes individually bring out the core pointers of this literature- "LinkedIn" and "Personal Branding" and "Job Search". "Branding" which audiences still relate to tangible products have slowly seeped into individuals. Individuals are making informed decisions and suitable actions in order to attract the attention of a specific set of people which in this case are job recruiters.

\section{What Is Linkedin}

LinkedIn, one of the leading social networking sites in the Internet world, designed with the intention of helping people get connected with each other professionally and benefit mutually in aspects pertaining to professional life such as:

a) Seeking Job Opportunities

b) Seeking suitable potential candidates for job opportunities

c) Increasing connections across organizations

d) Putting out professional accomplishments etc

Launched on May 5, 2003, by Reid Hoffman and his team, the intention behind LinkedIn was to successfully bring real-life professional relationships into the Internet and give users better access and opportunities to pursue the same and benefit from it. LinkedIn today stands at:
a) 690+ Million Members
b) $50 \mathrm{M}+$ Companies
c) $20 \mathrm{M}+$ Jobs
d) $36 \mathrm{~K}+$ Skills
e) $90 \mathrm{~K}+$ Schools
f) $280 \mathrm{~B}+$ Knowledge Materials

As per Karaduman (2013): The effect of social media on personal branding efforts of top-level executives: Procedia Social and Behavioral Sciences 99 ( 2013 ); 47, social media users posting relevant professional content on any social networking sites can be divided into three categories:

a) Casual Readers: Non-active users who use social media to read and do not share anything 
b) Reactive Authors: Users who share content as a reply to anything they wish to

c) Active Authors: Users who do not to stick to one specific kind of content to post

d) Proactive Authors: Users who create new ideas, put new agendas on the table

LinkedIn has launched a Premium, paid subscription, which enables users to be privy to premium services than normal users, such as-
a) Who viewed my profile
b) No. of visitors to my profile
c) Resume Generation
d) Access to $100 \mathrm{~s}$ of ofcourse and others

\section{Job Search Today}

The advent of digital age has opened up multiple avenues for all the stakeholders involved in job recruitment. Once limited to Recommendations and Newspaper advertisements or necessary examinations, job recruiters are employing the use of Job sites like naukri.com, monster.com etc to fulfill their manpower requirements. Recruiters believe it reduces overall Cost-To-Hire for them. Job seekers, do not mind being active on the above mentioned sites as it helps them being updated on the latest job openings.

Apart from these sources, there are two more major sources of Job Recruitments-

\section{a) Referrals b) Campus Recruitment Drives}

While the world has become increasingly smaller, companies seldom incentivize their employees if their referred candidates are hired by the organization. Such successful programs make In-House employees a major stakeholder in the process of recruitment too.

With the motivation of finding potential highly committed candidates, companies worldwide have continued to hire college graduates into their organizations in suitable roles.

Additionally, in areas of high workforce population density, some companies use "Walk-In Interviews" as a source of recruitment.

\section{Research Questions}

In order to initiate a comprehensive understanding towards LinkedIn, Job search and Personal Branding, we conducted primary research amongst the target audience. The target audience was divided into two groups-

a) Job Seekers- Students and Employed Professionals

b) Recruiters- HR Business Partners, Talent Acquisition Managers etc.

Both the groups had different questionnaires and were instructed to fill the same. The intent behind circulating different sets of questions and dividing them at the first place was to do a comparative study between the two parties.

\section{Survey 1: Job Seekers}

1) What is your primary motivation behind using LinkedIn?

The motive behind this particular question was to understand the psyche of the respondents. Understanding that not everyone is seeking jobs and there are other uses of LinkedIn, in order to quantify the same the following options were provided:

a) To attract the attention of recruiters and explore job openings

b) To maintain online professional relevance

c) To be in touch with latest biz and tech news

2) Since how long are you having a LinkedIn account, approximately?

This question aims to identify the average of the LinkedIn accounts of the respondents. It has been done to establish relation, if any, between the age of the account and successful job recruitment. To establish the same the following options were provided:

a) 1 Month b) 6 Months c) 1 Year d) > 1 Year e) Not an User

3) How many connections do you have on LinkedIn?

Since the underlying concept of LinkedIn is to connect with suitable people, No. of Connections is an important parameter which checks the effectiveness of a person's personal branding efforts which results in being hired for a job. To understand the same the below options were asked:

a) $500+$ b) $300+$ c) $100+$ d) Not an User

4) How will you define your LinkedIn activity?

This question has been asked to understand the basic need and to analyse the activities of the respondents on LinkedIn. To know the same, the following options were provided to choose from:

a) I read the latest updates and usually do not share anything.

b) I put my views on new agendas and trending topics proactively

c) I share different kinds of content with no fixed topic

d) I share content only as a reply to subject I am interested in

e) I do not use LinkedIn at all

5) How often do you share content on LinkedIn, approximately

Divulging more into understanding if the respondents are "Active" or "Passive" users of LinkedIn, this question was asked and to know the same the following options were given:
a) Once in a day
b) Once in Two Days
c) Once A Week
d) Once a Month
e) I do not share content on LinkedIn 
6) How often do you update your LinkedIn profile, approximately

Digging deeper to understand the nature of LinkedIn users, via the understated options given we tried to comprehend the same.

a) Once in a day

b) Once in Two Days

c) Once A Week

d) Once a Month

e) I do not share content on LinkedIn

7) How would you rate the below-mentioned sections of your LinkedIn Profile?

Notable sections of a LinkedIn profile includes:
a) About
b) Work experience
c) Skills, Endorsement and Recommendations Section
d) Accomplishments and Interests
e) Developing connections
f) Content

To measure the importance of these sections individually, we asked the respondents to choose one of the following for each section:

a) Least Important

b) Somewhat Important

c) Very Important

8) How would you rate the below-mentioned Profile parameters while accepting/ sending connection requests?

To understand the psychology behind accepting and sending connection requests from/ to 2 nd, 3 rd degree connections, like the previous questions, certain parameters were stated based on which the respondents had to choose either of- "Least Important", "Somewhat Important", "Most Important".

The parameters were:

a) Profile Photograph

b) About Section

c) Current Job Role/ Post

d) Current or Previous Organisation/ Institute

e) Past Content shared on LinkedIn

f) No. of Endorsements on Skills

g) No. of Recommendations

h) No. of Mutual Connections

i) No. of Connections

j) Resume

9) What kind of content do you like to read/ share on LinkedIn?

To understand the kind of usability behaviour respondents exhibit, this particular questions explores the type of content they like to use over LinkedIn:
a) Business Oriented
b) Technology Oriented
c) Personal Experiences
10) Are you seeking job opportunities presently?

In order to understand correlation, if any, between activity over LinkedIn and intent of seeking job opportunities, the following question was asked with two options:

a) Yes b) No

11) While applying for a job on LinkedIn, how would you rate the below-mentioned parameters?

While a candidate is seeking suitable opportunities over LinkedIn, the decision to apply for one while ignoring others is based on certain psychological decisions. To understand the same, the following parameters were provided with three options to rate them:

"Least Important", "Somewhat Important". "Most Important". The parameters were:
a) Organization
b) Job Profile
c) Recruiters' Personal Profile
12) Of the below mentioned, which one do you think will generate more job opportunities?
To understand the preferential choice between LinkedIn and other job seeking opportunities, the above question was asked and the below stated choices were given:
a) Job sites like Monster.com, Naukri.com, Indeed.com
b) LinkedIn
13) Do you use LinkedIn Premium?

The following question was asked with the intent to have an idea about no. of paid Premium users of LinkedIn.

The respondents had to answer in either: a) Yes b) No.

14) Do you agree with the following statements?

In continuation to the above question asked on LinkedIn premium, the following statements were put forward and respondents had to reply to them either in "Yes", "No" or "Never Used in Premium".

The statements are-

a) LinkedIn Premium has helped me connect with suitable connections

b) LinkedIn Premium has given me good job insights

c) LinkedIn Premium has increased the visibility and outreach of my posts

d) LinkedIn Premium has helped me connect with people other than my connections

e) LinkedIn Premium has helped me with its learning courses and certifications

15) Please respond to the following statements

The following statements were asked to have an overall detailed impression about respondents' LinkedIn profile. Respondents had to reply to them individually in "Yes", "No". The statements are-

a) I have received more connection request when I post my own original content

b) I have got recommendations on LinkedIn

c) I have written recommendations for someone on LinkedIn

d) My skills have been endorsed by others on LinkedIn 
e) I have endorsed others skills on LinkedIn

f) I have applied for a job in LinkedIn before

g) I have received a job referral on LinkedIn

h) I have bagged a job via referral on LinkedIn

i) I have applied for a job on LinkedIn and got the job

j) After applying for a job, I have personally contacted the recruiter

k) I have applied for job in other sources such as job sites, in parallel to applying on LinkedIn?

1) I have received benefits other than job referrals, such as Assistance on Work, Certifications, LinkedIn Premium giveaway etc from my connections

m) If I do not have new content, I comment on insightful posts to maintain relevance

\section{Survey 2: Recruiters}

Parallely, another questionnaire was circulated amongst the set of stakeholders- Recruiters, Talent Acquisition Specialists, HR Managers etc who actively use LinkedIn and other mediums to hire job seekers. Via the questionnaire, the intent is to gain insights from the data and draw conclusions accordingly.

1. How long are you recruiting candidates?

To understand the various demographic details of the respondents, the following questions and the respective options were given:

2. What is your Gender?

i) Female ii) Male iii) Prefer not to say

3. What is your age?

i) 18-21 ii) 21- 26 iii) 27 and Above

4. What industry do you work in?

i) FMCG/ FMCD ii) IT/ ITES iii) Central Government iv)

Education v) Banking vi) Automobile vii) Civil Aviation

The following questions were asked to understand the LinkedIn activity of the recruiters.

5. How many connections do you have on LinkedIn?

i) $500+$ ii) $300+$ iii) $100+$

6. How frequently do you post job openings on LinkedIn, approximately?

i) At Least once a month ii) Once in two months iii) Once in six months

7. How would you rate the importance of the belowmentioned parameters of a candidate's

LinkedIn Profile while evaluating his/ her candidacy?

- $\quad$ Profile Photograph

- About Section

- Current Job Role/ Post

- Current or Previous Organisation/ Institute

- Resume

- $\quad$ Past Content shared on LinkedIn

- $\quad$ No. of Endorsements on Skills

- No. of Recommendations

- No. of Connections
The above stated profile parameters had to be rated individually as:

i) Least Important ii) Somewhat Important iii) Very Important

8. Please provide your answers to these questions

- $\quad$ Do you use LinkedIn for recruiting candidates?

- Does your organization use tools like LinkedIn Recruiter?

- Do you think LinkedIn Recruiter gives the best candidates?

- Do you trust LinkedIn as the sole source of recruitment?

- Do you receive frequent personal messages on LinkedIn for job openings?

- Do you trust overall a candidate's LinkedIn Profile over his/ her Resume?

- Have you ever selected candidates who applied for a job from LinkedIn?

- Do you use LinkedIn Premium?

The above stated questions had to be answered individually as: i) Yes ii) No

Table-1

Demographic Profile of Participants who are seeking jobs over LinkedIn

\begin{tabular}{|c|c|c|c|}
\hline $\begin{array}{c}\text { Demographic } \\
\text { Variable }\end{array}$ & Value & $\mathbf{n}$ & $\%$ \\
\hline & Male & 174 & $69.90 \%$ \\
\hline Gender & Female & 75 & $30.10 \%$ \\
\hline Total & & 249 & $100.00 \%$ \\
\hline & & & \\
\hline & $18-21$ & 81 & $32.50 \%$ \\
\hline Age & $22-26$ & 124 & $49.80 \%$ \\
\hline & $>27$ & 44 & $17.70 \%$ \\
\hline Total & & 249 & $100.00 \%$ \\
\hline & & 93 & $37.30 \%$ \\
\hline & Under Graduate Student & 93 & $33.70 \%$ \\
\hline Background & Post Graduate Student & 84 & $28.90 \%$ \\
\hline & Working Professional & 72 & $99.90 \%$ \\
\hline Total & & 249 & \\
\hline & & & \\
\hline
\end{tabular}

Table-2

Demographic Profile of Participants who are recruiters

\begin{tabular}{|c|c|c|c|}
\hline $\begin{array}{c}\text { Demographic } \\
\text { Variable }\end{array}$ & Value & $\mathbf{n}$ & $\%$ \\
\hline \multirow[t]{2}{*}{ Gender } & Male & 38 & $66.70 \%$ \\
\hline & Female & 19 & $33.30 \%$ \\
\hline Total & & 57 & $100.00 \%$ \\
\hline & & & \\
\hline \multirow[t]{2}{*}{ Age } & $21-26$ & 14 & $24.60 \%$ \\
\hline & $>27$ & 43 & $75.40 \%$ \\
\hline \multirow[t]{4}{*}{ Total } & & 57 & $100.00 \%$ \\
\hline & FMCG/FMCD & 4 & $7 \%$ \\
\hline & IT/ ITES & 18 & $31.60 \%$ \\
\hline & Central Government & 2 & $3.50 \%$ \\
\hline \multirow[t]{5}{*}{ Industry } & Education & 5 & $8.80 \%$ \\
\hline & Banking & 2 & $3.50 \%$ \\
\hline & Automobile & 3 & $5.30 \%$ \\
\hline & Civil Aviation & 1 & $1.80 \%$ \\
\hline & Others & 22 & $38.60 \%$ \\
\hline Total & & 57 & $100 \%$ \\
\hline
\end{tabular}




\section{4. $\quad$ Results}

To determine the relationship between the various categorical variables to come into a conclusion we used Cross Tabs and subsequently applied Chi-Square tests to determine the significance of any relationship. For reference we have considered $95 \%$ as the confidence interval.

In the below stated, we are considering the results we have got and analysis we have made from the responses obtained from job seekers.

Null Hypothesis: There is a significant correlation between the two variables

Alternate Hypothesis: There is no significant correlation between the two variables.

From the results obtained above we can tabulated data and additionally we can see that $\mathrm{p}(0.00)<0.05$, hence there is a significant correlation between "What the respondent is doing currently i.e. Undergraduate/ Postgraduate Student/ Working Professional and "How long they are having a LinkedIn account".

On similar lines we have found out significant correlation between "What are you doing Currently" and the understated categorical variables-

What are you doing currently vs

1. How long do you have a LinkedIn account?

$$
\text { crosstab }
$$
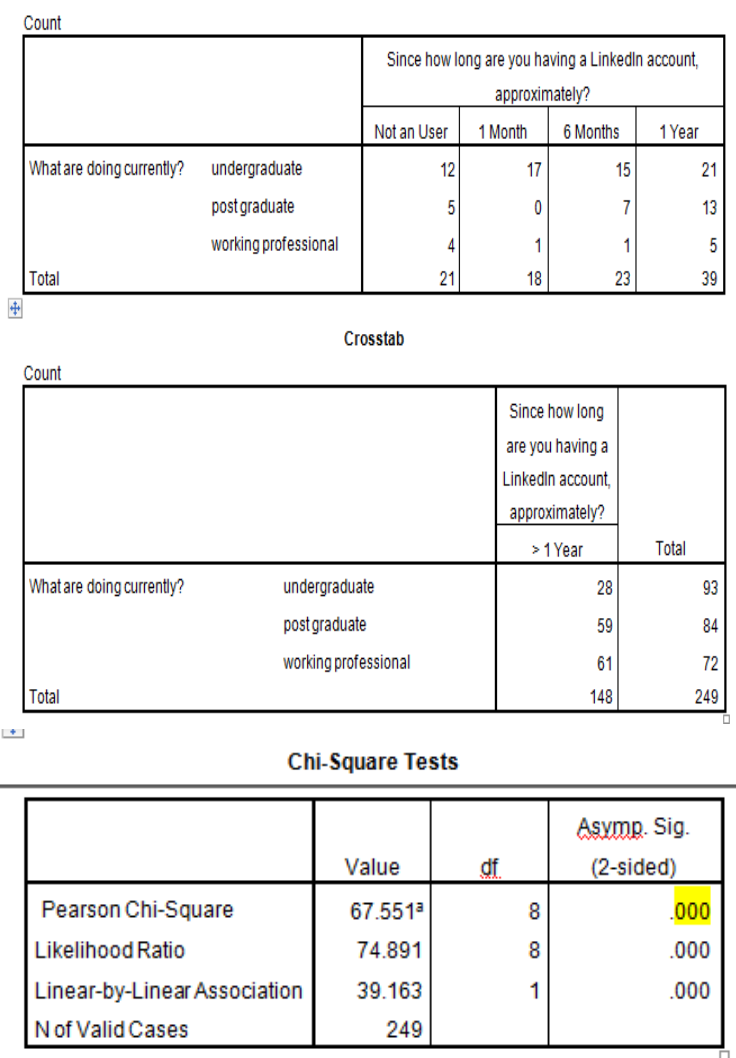

a. 0 cells $(.0 \%)$ have expected count less than 5 . The minimum expected countis 5.20 .
2. How many connections on LinkedIn? Crosstab

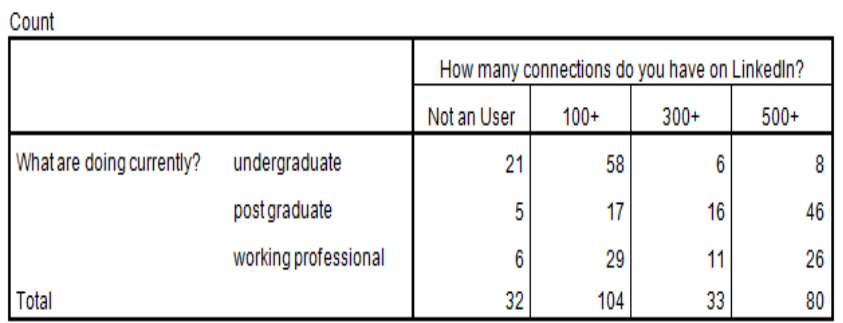

Chi-Square Tests

\begin{tabular}{|l|r|r|r|}
\hline & \multicolumn{1}{|c|}{ Value } & df. & \multicolumn{1}{c|}{$\begin{array}{c}\text { Asymp. Sig. } \\
\text { (2-sided) }\end{array}$} \\
\hline Pearson Chi-Square & $65.279^{\mathrm{a}}$ & 6 & .000 \\
Likelihood Ratio & 70.691 & 6 & .000 \\
Linear-by-Linear Association & 26.185 & 1 & .000 \\
N of Valid Cases & 249 & & \\
\hline
\end{tabular}

3. How often do you share content on Linkedin? Crosstab

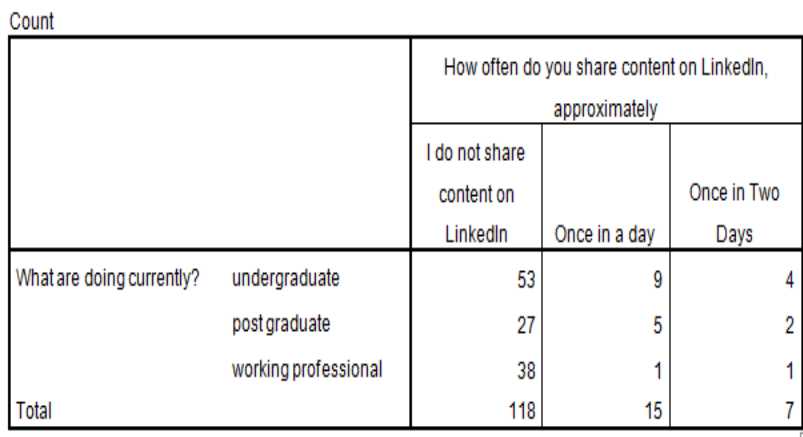

Crosstab

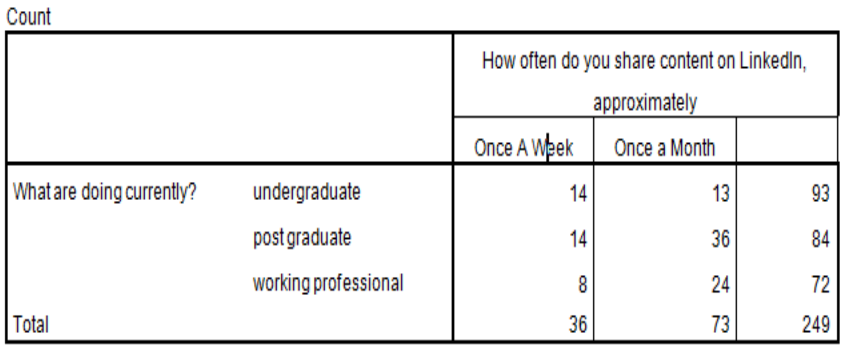

\begin{tabular}{|l|r|r|r|}
\hline & Chi-Square Tests \\
& \multicolumn{1}{|c|}{ Value } & df & $\begin{array}{c}\text { Asymp. Sig. } \\
\text { (2-sided) }\end{array}$ \\
\hline Pearson Chi-Square & $26.283^{\mathrm{a}}$ & 8 & .001 \\
Likelihood Ratio & 28.722 & 8 & .000 \\
\hline Linear-by-Linear Association & 4.432 & 1 & .035 \\
N of Valid Cases & 249 & & \\
\hline
\end{tabular}

a. 4 cells $(26.7 \%)$ have expected countless than 5 . The minimum expected countis 2.02 . 
4. How often do you update your LinkedIn profile? Crosstab

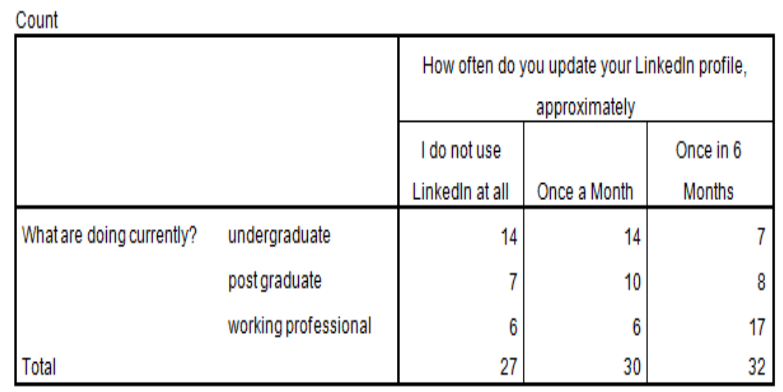

Crosstab

Count

\begin{tabular}{|c|c|c|c|}
\hline & & $\begin{array}{c}\text { How often do you } \\
\text { update your Linkedln } \\
\text { profile, approximately }\end{array}$ & \\
\hline & & $\begin{array}{c}\text { As per requirements, } \\
\text { accomplishments, } \\
\text { changes } \\
\end{array}$ & Total \\
\hline What are doing currently? & undergraduate & 58 & 93 \\
\hline & postgraduate & 59 & 84 \\
\hline & working professional & 43 & 72 \\
\hline Total & & 160 & 249 \\
\hline
\end{tabular}

Chi-Square Tests

\begin{tabular}{|l|r|r|r|}
\hline & \multicolumn{1}{|c|}{ Value } & \multicolumn{1}{c|}{ df. } & \multicolumn{1}{c|}{$\begin{array}{c}\text { Asymp. Sig. } \\
\text { (2-sided) }\end{array}$} \\
\hline Pearson Chi-Square & $13.962^{\mathrm{a}}$ & 6 & .030 \\
Likelihood Ratio & 13.052 & 6 & .042 \\
Linear-by-Linear Association & 1.313 & 1 & .252 \\
Nof Valid Cases & 249 & & \\
\hline
\end{tabular}

5. My skills has been endorsed by others Crosstab

\begin{tabular}{|c|c|c|c|c|}
\hline & & \multicolumn{2}{|c|}{$\begin{array}{c}\text { My skills have been endorsed by } \\
\text { others on Linkedln }\end{array}$} & \multirow[b]{2}{*}{ Total } \\
\hline & & No & Yes & \\
\hline \multirow[t]{2}{*}{ What are doing currently? } & undergraduate & 58 & 35 & 93 \\
\hline & postgraduate & 30 & 54 & 84 \\
\hline & working professional & 26 & 46 & 72 \\
\hline Total & & 114 & 135 & 249 \\
\hline
\end{tabular}

\begin{tabular}{|l|r|r|r|}
\hline & \multicolumn{1}{|c|}{ Chi-Square Tests } \\
& \multicolumn{1}{|c|}{ Value } & \multicolumn{1}{c|}{ df. } & $\begin{array}{c}\text { Asymp. Sig. } \\
\text { (2-sided) }\end{array}$ \\
\hline Pearson Chi-Square & $16.447^{\mathrm{a}}$ & 2 & .000 \\
Likelihood Ratio & 16.557 & 2 & .000 \\
Linear-by-Linear Association & 12.318 & 1 & .000 \\
Nof Valid Cases & 249 & & \\
\hline
\end{tabular}

6. Received Job Referral

Crosstab
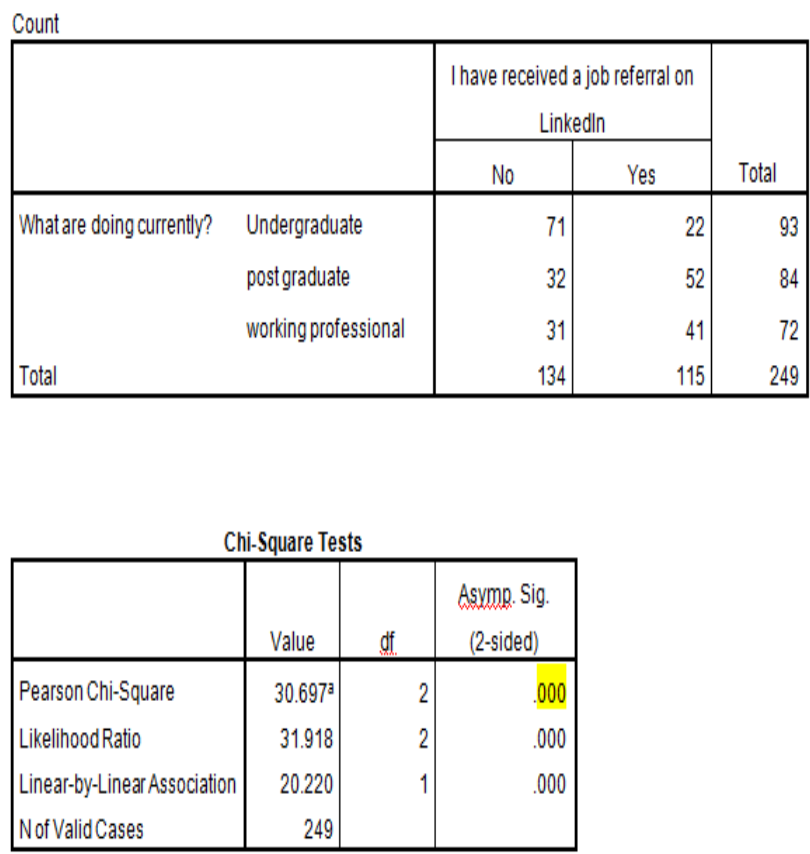

7. After applying job, I personally contacted the recruiter

Crosstab
\begin{tabular}{|ll|r|r|r|}
\hline \multicolumn{1}{|c|}{ Count } & \multicolumn{2}{c|}{ After applying for a job, I have } & \\
& & \multicolumn{2}{c|}{ personally contacted the recruiter } \\
\cline { 3 - 4 } & & No & Yes & Total \\
\hline What are doing currently? & undergraduate & 82 & 11 & 93 \\
& post graduate & 60 & 24 & 84 \\
& working professional & 56 & 16 & 72 \\
& & 198 & 51 & 249 \\
\hline
\end{tabular}




\section{Chi-Square Tests}

\begin{tabular}{|l|r|r|r|}
\hline & \multicolumn{1}{|c|}{ Value } & \multicolumn{1}{c|}{$\mathrm{df}$} & $\begin{array}{c}\text { Asymp. Sig. } \\
\text { (2-sided) }\end{array}$ \\
\hline Pearson Chi-Square & $7.785^{\mathrm{s}}$ & 2 & .020 \\
Likelihood Ratio & 8.097 & 2 & .017 \\
Linear-by-Linear Association & 3.241 & 1 & .072 \\
Nof Valid Cases & 249 & & \\
\hline
\end{tabular}

8. Bagged a job via referral on LinkedIn crosstab

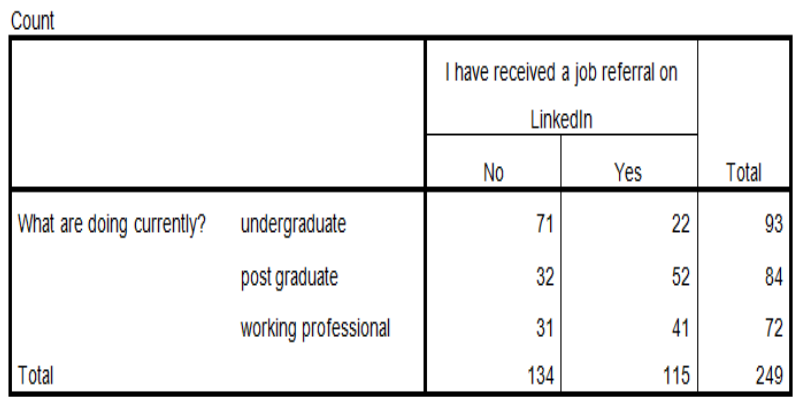

\begin{tabular}{|c|c|c|c|}
\hline \multicolumn{4}{|c|}{ Chi-Square Tests } \\
\hline & Value & $\mathrm{df}$ & $\begin{array}{c}\text { Asymp. Sig. } \\
\text { (2-sided) }\end{array}$ \\
\hline Pearson Chi-Square & $30.697^{\mathrm{g}}$ & 2 & .000 \\
\hline Likelihood Ratio & 31.918 & 2 & .000 \\
\hline Linear-by-Linear Association & 20.220 & 1 & .000 \\
\hline N of Valid Cases & 249 & & \\
\hline
\end{tabular}

9. I have written recommendations for someone on LinkedIn

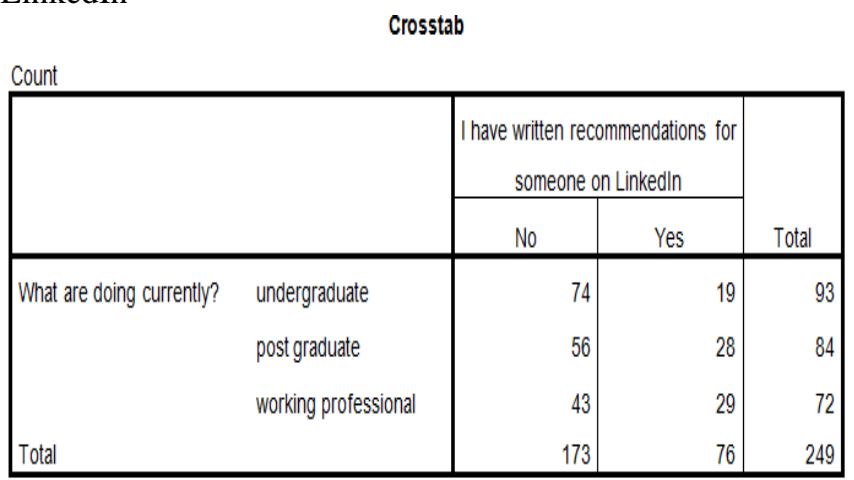

\begin{tabular}{|c|c|c|c|}
\hline \multicolumn{4}{|c|}{ Chi-Square Tests } \\
\hline & Value & $\mathrm{df}$ & $\begin{array}{c}\text { Asymp. Sig. } \\
\text { (2-sided) }\end{array}$ \\
\hline Pearson Chi-Square & $8.011^{\mathrm{s}}$ & 2 & .018 \\
\hline Likelihood Ratio & 8.200 & 2 & .017 \\
\hline Linear-by-Linear Association & 7.748 & 1 & .005 \\
\hline N of Valid Cases & 249 & & \\
\hline
\end{tabular}

10. Received other stuffs such as Certificates, Premium etc Crosstab Count

\begin{tabular}{|c|c|c|c|c|}
\hline & & \multicolumn{2}{|c|}{$\begin{array}{l}\text { I have received benefits other than } \\
\text { job referrals, such as Assistance } \\
\text { on Work, Certifications, Linkedln } \\
\text { Premium giveaway etc from my } \\
\text { connections }\end{array}$} & \multirow[b]{2}{*}{ Total } \\
\hline & & No & Yes & \\
\hline \multirow[t]{3}{*}{ What are doing currently? } & undergraduate & 70 & 23 & 93 \\
\hline & post graduate & 46 & 38 & 84 \\
\hline & working professional & 57 & 15 & 72 \\
\hline Total & & 173 & 76 & 249 \\
\hline
\end{tabular}

\begin{tabular}{|l|r|r|r|}
\hline & Chi-Square Tests \\
& \multicolumn{1}{|c|}{ Value } & \multicolumn{1}{c|}{$\mathrm{df}$} & $\begin{array}{c}\text { Asymp. Sig. } \\
\text { (2-sided) }\end{array}$ \\
\hline Pearson Chi-Square & $13.236^{\mathrm{s}}$ & 2 & .001 \\
Likelihood Ratio & 12.964 & 2 & .002 \\
Linear-by-Linear Association & .073 & 1 & .787 \\
Nof Valid Cases & 249 & & \\
\hline
\end{tabular}

In addition to the What the respondents are doing currently, we also did cross tabulation analysis between the "age of the respondents" vs-

1. Since how long have they been having a LinkedIn account?

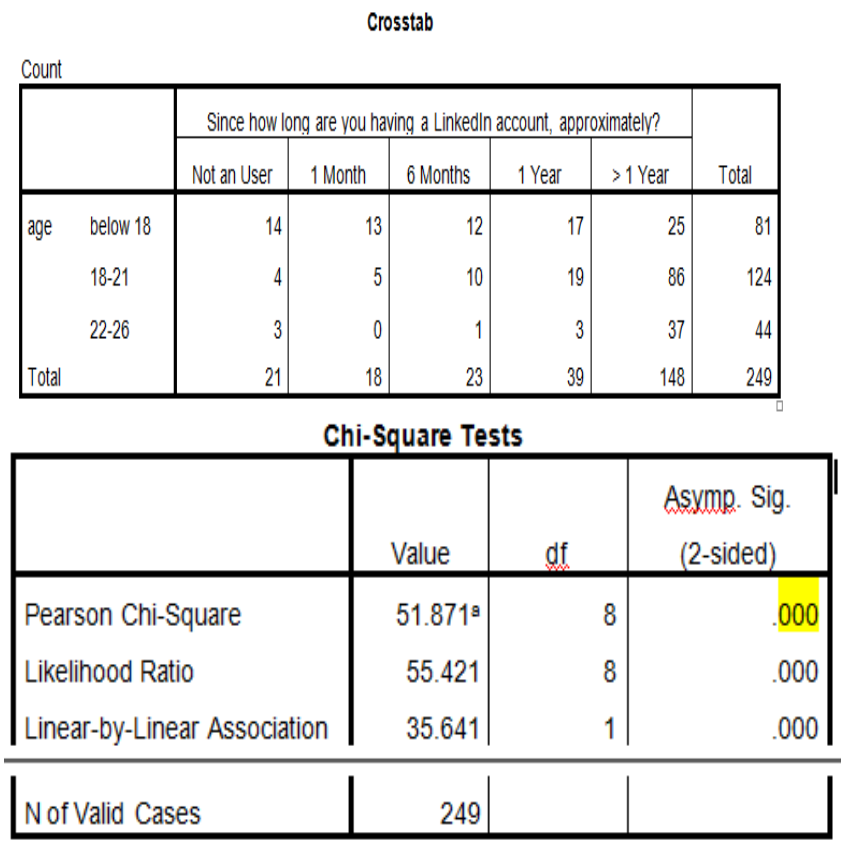


2. How many connections on LinkedIn? Crosstab

\begin{tabular}{|l|r|r|r|r|r|r|}
\hline & \multicolumn{2}{|c|}{ Count } & \multicolumn{2}{|c|}{ How many connections do you have on Linkedln? } & \multirow{2}{*}{} \\
\cline { 3 - 6 } & & Not an User & $100+$ & $300+$ & $500+$ & Total \\
\hline age & below 18 & 21 & 47 & 4 & 9 & 81 \\
& $18-21$ & 8 & 35 & 24 & 57 & 124 \\
& $22-26$ & 3 & 22 & 5 & 14 & 44 \\
Total & & 32 & 104 & 33 & 80 & 249 \\
\hline
\end{tabular}

3. Written recommendations for someone on LinkedIn Crosstab

\begin{tabular}{|ll|r|r|r|}
\hline & \multicolumn{2}{|c|}{$\begin{array}{c}\text { I have written recommendations for } \\
\text { someone on Linkedln }\end{array}$} & \multirow{2}{*}{ Total } \\
\cline { 3 - 4 } & No & Yes & 81 \\
\cline { 3 - 4 } & below 18 & 65 & 16 & 124 \\
& $18-21$ & 81 & 43 & 44 \\
& $22-26$ & 27 & 17 & 249 \\
\hline
\end{tabular}

\begin{tabular}{|l|r|r|r|}
\hline & \multicolumn{1}{|c|}{ Chi-Square Tests } \\
& \multicolumn{1}{|c|}{ Value } & df & $\begin{array}{c}\text { Asymp. Sig. } \\
\text { (2-sided) }\end{array}$ \\
\hline Pearson Chi-Square & $6.805^{9}$ & 2 & .033 \\
Likelihood Ratio & 7.104 & 2 & .029 \\
Linear-by-Linear Association & 5.939 & 1 & .015 \\
N of Valid Cases & 249 & & \\
\hline
\end{tabular}

4. How often do you update your LinkedIn profile?

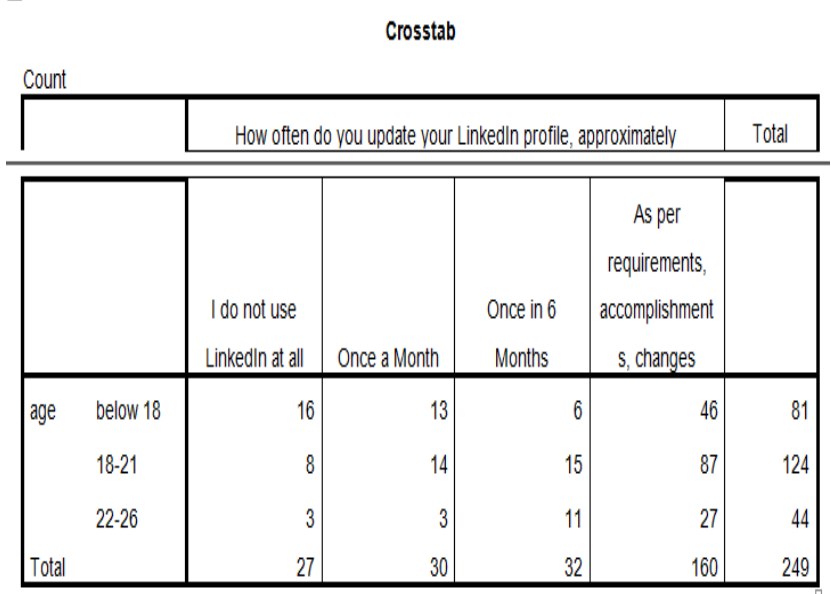

Chi-Square Tests

\begin{tabular}{|l|r|r|r|}
\hline & \multicolumn{1}{|c|}{ Value } & df & \multicolumn{1}{c|}{$\begin{array}{c}\text { Asymp. Sig. } \\
\text { (2-sided) }\end{array}$} \\
\hline Pearson Chi-Square & $19.336^{\mathrm{a}}$ & 6 & .004 \\
Likelihood Ratio & 17.977 & 6 & .006 \\
Linear-by-Linear Association & 6.045 & 1 & .014 \\
N of Valid Cases & 249 & & \\
\hline
\end{tabular}

5. Skills endorsed by others Crosstab

\begin{tabular}{|ll|r|r|r|}
\hline & \multicolumn{2}{|c|}{$\begin{array}{c}\text { My skills have been endorsed by } \\
\text { others on Linkedln }\end{array}$} & \multirow{2}{*}{ Total } \\
\cline { 3 - 4 } & No & Yes & \multicolumn{1}{|c|}{ T1 } \\
\cline { 3 - 4 } & below 18 & 48 & 33 & 124 \\
& $18-21$ & 48 & 76 & 44 \\
& $22-26$ & 18 & 26 & 249 \\
\hline
\end{tabular}

\begin{tabular}{|l|r|r|r|}
\hline & Chi-Square Tests \\
& Value & df & $\begin{array}{c}\text { Asymp. Sig. } \\
\text { (2-sided) }\end{array}$ \\
\hline Pearson Chi-Square & $8.847 \mathrm{~g}$ & 2 & .012 \\
\hline Likelihood Ratio & 8.861 & 2 & .012 \\
Linear-by-Linear Association & 5.727 & 1 & .017 \\
N of Valid Cases & 249 & & \\
\hline
\end{tabular}

6. Received a job referral

Crosstab

\begin{tabular}{|c|c|c|c|c|}
\hline & & \multicolumn{2}{|c|}{$\begin{array}{l}\text { I have received a job referral on } \\
\text { Linkedln }\end{array}$} & \multirow[b]{2}{*}{ Total } \\
\hline & & No & Yes & \\
\hline \multirow[t]{3}{*}{ age } & below 18 & 62 & 19 & 81 \\
\hline & $18-21$ & 55 & 69 & 124 \\
\hline & $22-26$ & 17 & 27 & 44 \\
\hline Total & & 134 & 115 & 249 \\
\hline
\end{tabular}

\section{Chi-Square Tests}

\begin{tabular}{|l|r|r|r|}
\hline & \multicolumn{1}{|c|}{ Value } & df & $\begin{array}{c}\text { Asymp. Sig. } \\
\text { (2-sided) }\end{array}$ \\
\hline Pearson Chi-Square & $25.378^{a}$ & 2 & .000 \\
Likelihood Ratio & 26.468 & 2 & .000 \\
Linear-by-Linear Association & 21.107 & 1 & .000 \\
N of Valid Cases & 249 & & \\
\hline
\end{tabular}


7. Personally contacted the recruiter after applying for job

\section{Crosstab}

\begin{tabular}{|ll|r|r|r|}
\hline & \multicolumn{2}{|c|}{$\begin{array}{c}\text { After applying for a job, I have } \\
\text { personally contacted the recruiter }\end{array}$} & \multirow{2}{*}{ Total } \\
\cline { 3 - 4 } & No & Yes & \multicolumn{1}{|c|}{ Tes } \\
\cline { 3 - 4 } & below 18 & 72 & 9 & 81 \\
& $18-21$ & 94 & 30 & 124 \\
& $22-26$ & 32 & 12 & 44 \\
Total & & 198 & 51 & 249 \\
\hline
\end{tabular}

\begin{tabular}{|c|c|c|c|}
\hline & Value & $\mathrm{df}$ & $\begin{array}{c}\text { Asymp. Sig. } \\
\text { (2-sided) }\end{array}$ \\
\hline Pearson Chi-Square & $6.662^{\mathrm{a}}$ & 2 & .036 \\
\hline Likelihood Ratio & 7.198 & 2 & .027 \\
\hline Linear-by-Linear Association & 5.726 & 1 & .017 \\
\hline N of Valid Cases & 249 & & \\
\hline
\end{tabular}

In addition to that, some important which we must consider before coming to conclusion-

What are doing currently? * Rate the Skills, Endorsement and Recommendations Section for your own profile Crosstabulation

\begin{tabular}{|ll|r|r|r|}
\hline \multirow{5}{*}{ Count } & \multicolumn{3}{|c|}{$\begin{array}{c}\text { Rate the Skills, Endorsement and } \\
\text { Recommendations Section for your own profile }\end{array}$} \\
\cline { 2 - 5 } & & Somewhat & \\
& & Least Important & Important & Very Important \\
\hline What are doing currently? & undergraduate & 7 & 31 & 55 \\
& post graduate & 3 & 30 & 51 \\
& working professional & 5 & 23 & 44 \\
& & 15 & 84 & 150 \\
\hline
\end{tabular}

This particular table proves that irrespective of what their background is, respondents consider being endorsed and getting recommendations to their profile as very important. What are doing currently? * Current or Previous Organisation/ Institute for connecting Crosstabulation

\begin{tabular}{|ll|r|r|r|}
\hline \multirow{2}{*}{ Count } & \multicolumn{3}{|c|}{ Current or Previous Organisation/ Institute for } \\
& & \multicolumn{3}{|c|}{ connecting } \\
\cline { 2 - 5 } & & \multicolumn{1}{|c|}{ Somewhat } & \\
& Least Impoortant & Important & Very Important \\
\hline What are doing currently? & undergraduate & 7 & 43 & 43 \\
& post graduate & 3 & 30 & 51 \\
& working professional & 2 & 19 & 51 \\
& & 12 & 92 & 145 \\
\hline
\end{tabular}

Similarly, this tabled data proves that while connecting with a 2nd/ 3rd Degree connection, an user does consider the potential connection's background i.e. educational institute/ organisation.

Are you seeking job opportunities presently? * which one do you think will generate more job opportunities? Crosstabulation

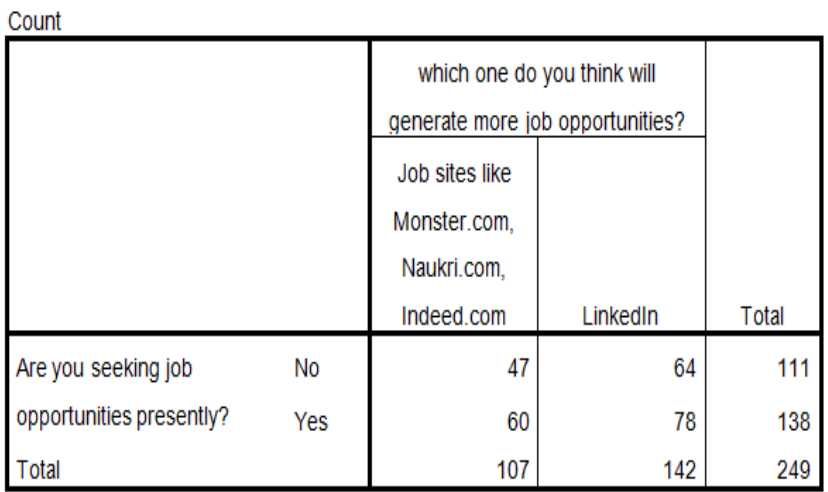

Considering all the factors, the respondents cited that LinkedIn is a better platform to see job opportunities than the quintessential job portals like Naukri,Indeed etc.

In terms of recruiters, let us first consider the assumption that all of them are LinkedIn users and know the functionalities well. Secondly, we are putting out some tabulated data below to give a perspective on recruiters' take on LinkedIn.

Age * Does your organization use tools like Linkedln Recruiter? Crosstabulation

\begin{tabular}{|c|c|c|c|c|}
\hline \multicolumn{5}{|c|}{ Count } \\
\hline & & \multicolumn{2}{|c|}{$\begin{array}{c}\text { Does your organization use tools } \\
\text { like Linkedln Recruiter? }\end{array}$} & \multirow[b]{2}{*}{ Total } \\
\hline & & No & Yes & \\
\hline & $21-26$ & 5 & 9 & 14 \\
\hline & 27 and Above & 20 & 22 & 42 \\
\hline Total & & 25 & 31 & 56 \\
\hline
\end{tabular}

So organizations do use LinkedIn and its functionality LinkedIn recruiter to seek candidates.

Age * Do you think Linkedln Recruiter gives the best candidates? Crosstabulation

\begin{tabular}{|c|c|c|c|c|}
\hline & & \multicolumn{2}{|c|}{$\begin{array}{l}\text { Do you think Linkedln Recruiter } \\
\text { gives the best candidates? }\end{array}$} & \multirow[b]{2}{*}{ Total } \\
\hline & & No & Yes & \\
\hline \multirow[t]{2}{*}{ Age } & $21-26$ & 7 & 7 & 14 \\
\hline & 27 and Above & 23 & 19 & 42 \\
\hline Total & & 30 & 26 & 56 \\
\hline
\end{tabular}

However the respondents are undecided whether it gives them the best candidates or not. 


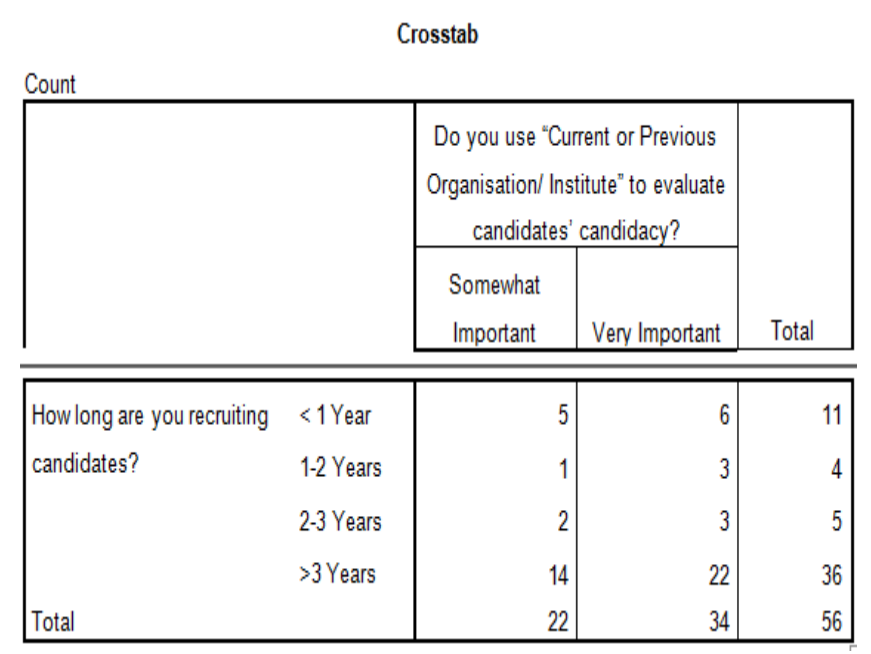

Just like the jobs seekers, the recruiters keep a tab on candidates' educational/ professional background while considering them for a position.

\section{Conclusions}

The Influence of Employer branding and Employees' personal branding on Corporate Branding and Corporate Reputation by Potgieter \& Doubell, says that- "Personal branding, in its broadest form, can be defined as a perception maintained by somebody else about an individual, the meaningful perceptions about the values and qualities of an individual, and a reflection of what an individual believes in and is expressed by what they do and how they do it (Rampersad, 2015; 2009.)" . It is understandably very clear that while being scrutinized for a job, an individual's personal brand is a testament to his/ her character.

In terms of social media and LinkedIn, based on the above results obtained, some of the activities and factors, which can determine a job seekers' personal brand are-

- $\quad$ Age of LinkedIn account

- $\quad$ Age of the LinkedIn user

- Educational and other background of the user

- Users' activity on LinkedIn account which includes type of content shared, updates made etc.

- No. and type of connections one has in their LinkedIn account.

The result of personal branding over LinkedIn includes:

- Receiving job referrals

- Receiving personalized endorsements

- Receiving news, certifications and premium giveaways from connections

From the results obtained we can say that from all the parameters listed, there are some which stand out and give us a clear picture towards the correlations between LinkedIn, Personal Branding and Job prospects.

We can safely conclude that job seekers consider establishing a personal brand over LinkedIn to be beneficial and for the same they must be active on LinkedIn. As obtained from the result, activeness over LinkedIn is measured by the number i.e. between 100-500 and the diversity of connections one has, how regularly the profile is updated. Apart from building it, in order to build a personal brand it is equally important to interact with your network and that can be done by sharing relevant engaging content on regular intervals, endorsing and recommending team members.

If a particular user follows all of the above stated diligently at least for an year or so, as a reaction, he/she may receive job referrals over LinkedIn from the network he/ she has established, which can be converted into an opportunity.

While we fully consider that the educational/ professional background of a job seeker matters, we cannot ignore the fact that age of the candidate does have an impact with his/her conduct over LinkedIn. This proves that while the above mentioned parameters' effect on Personal Branding is proved and tabulated, personal branding and activities determining it, is subjective in nature and that more factors can be added to this in near future.

\section{Limitations \& Scope}

As stated in the opening statements of this paper, two surveys were conducted, one for the job seekers which has been discussed at length. However the other survey pertaining to recruiters has not been discussed. The reason for the same is that we were not able to find any significant relationship between any of the categorical variables. In comparison to the job seekers, the no. of respondents who are recruiters is very low and we believe that, the data is not a true reflection of their behaviour considering they were trying to be politically correct in their answers.

We believe, with greater sample size and without any physical/ mental barriers, much better analysis can be done.

Apart from exploring the respondents' pick, future study can find out the effect of paid subscription plan- "LinkedIn Premium" over Personal Branding and job prospects. Perhaps a detailed study on LinkedIn Premium could point out the differences between the effects of Personal Branding efforts over a normal one and premium one.

\section{References}

[1] 'Ctrl, Alt, Delete'- Mitch Joel

[2] 'Distinguish yourself and excel in the profession'Annabelle Reitman and Caitlin Williams

[3] "LinkedIn or Left Out"- Neal Schaffer

[4] The effect of social media on personal branding efforts of top-level executives: Procedia - Social and Behavioral Sciences 99 ( 2013 ) - Karaduman

[5] The Influence of Employer branding and Employees' personal branding on Corporate Branding and Corporate Reputation- Potgieter \& Doubell. 